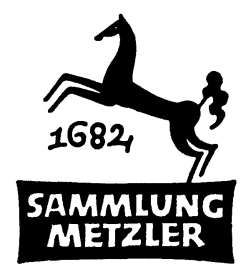

REALIEN ZUR LITERATUR

ABT. E:

POETIK 


\section{Die literarische Utopie}

2., neubearbeitete Auflage

ERSCHIENEN IM DREIHUNDERTSTEN JAHR DER J. B. METZLERSCHEN VERLAGSBUCHHANDLUNG STUTTGART 
CIP-Kurztitelaufnahme der Deutschen Bibliothek

Biesterfeld, Wolfgang:

Die literarische Utopie / Wolfgang Biesterfeld. -

2., neubearb. Aufl. - Stuttgart: Metzler, 1982.

(Sammlung Metzler; M 127: Abt. E, Poetik)

ISBN 978-3-476-12127-1

NE: GT

ISBN 978-3-476-12127-1

ISBN 978-3-476-04086-2 (eBook)

DOI $10.1007 / 978-3-476-04086-2$

M 127

(C) Springer-Verlag GmbH Deutschland 1982

Ursprünglich erschienen bei J. B. Metzlersche Verlagsbuchhandlung und Carl Ernst Poeschel Verlag GmbH in Stuttgart 1974/1982 


\section{INHALT}

Verzeichnis der wichtigsten Literatur . . . . . . . . IX

I. Einleitung $\ldots \ldots \ldots \ldots \ldots \ldots \ldots \ldots \ldots \ldots \ldots \ldots \ldots$

1. Differenzierungen im Begriff »Utopie « ............. 1

2. Probleme der Typologie

Definitionen - Die Utopie als Textsorte und in den Textsorten - Konstituenten der Utopie ................... 8

3. Wurzeln im Mythos und das Märchen vom Schlaraffenland ... 22

4. Beziehungen zu Architektur und Städtebau . . . . . . . . . 27

II. Platons Politeia und die Klassische Utopie $\ldots \ldots \ldots \quad 30$

1. Platons Staatsentwürfe und ihre Rezeption $\ldots \ldots \ldots \ldots \ldots \quad 30$

2. Von der Antike zum Spätmittelalter $\ldots \ldots \ldots \ldots \ldots \ldots . \quad 33$

3. Verzeichnis der wichtigsten Utopien des 16. und 17. Jahrhunderts ............................ 37

4. Morus, Campanella, Andreae, Bacon ............ 41

5. Verzeichnis der wichtigsten Utopien des 18. Jahrhunderts ... 48

6. Probleme und Formen der Utopie im 18. Jahrhundert Das Epochenproblem - Robinson - Die Insel Felsenburg Edle Wilde, neue Welten - Gelehrtenrepubliken - Wunderbare Reisen .............................. 53

III. FürstensPIEgEL $\ldots \ldots \ldots \ldots \ldots \ldots \ldots \ldots \ldots \ldots \ldots \ldots$

1. Typologie und Übersicht $\ldots \ldots \ldots \ldots \ldots \ldots \ldots \ldots .65$

2. Xenophons $»$ Kyrupädie $\ldots \ldots \ldots \ldots \ldots \ldots \ldots \ldots, 68$

3. Fénelon und seine Nachfolger ................ 69

4. Albrecht v. Hallers Staatsromane und Wielands "Goldner Spiegel ${ }^{\prime} \ldots \ldots \ldots \ldots \ldots \ldots \ldots \ldots \ldots \ldots \ldots, 72$

IV. UTOPIEN DES 19. und 20. JAHRHUNDERTS $\ldots \ldots \ldots \ldots .76$

1. Das 19. Jahrhundert. Überblick zu den englischen Utopien des 19. Jahrhunderts ..................... 76

2. Englische Utopien und Anti-Utopien seit $1900 \ldots \ldots \ldots \ldots .82$

3. Die deutsche Utopie seit 1800. Einleitung und Versuch einer Bibliographie ...........................

4. Formen der deutschen Utopie im 20. Jahrhundert Hauptmann - Hesse - Werfel - Jünger - Enzensberger Dürrenmatt ....................... 96

5. Exkurs: Utopien in anderen Literaturen des 20. Jahrhunderts .. 103

6. Science Fiction ............................ 107

ANHANG: Auswahlbibliographie zu Utopie und Science Fiction im Literaturunterricht

Register 


\section{VORWORT ZUR 2. AUfLAGE}

Es ist mittlerweile nicht leichter geworden, ein großes Thema in kleinem Rahmen darzustellen, mag auch die Utopienproduktion nachgelassen und die Utopienforschung zugenommen haben. Unser Versuch hat wiederum die Gestalt einer kommentierten Bibliographie, wobei einerseits der Anschluß an die Gegenwart gesucht wird, andererseits aber zahlreiche Texte der primären und sekundären Literatur ausgeschieden werden mußten, wofür der Grund in einer noch strengeren Handhabung des Utopie-Begriffs zu sehen ist: Es geht um die im Text manifestierte Sozial-Utopie. In Zuspruch und Kritik zur 1. Auflage hat diese bewußte Begrenzung zu Mißverständnissen geführt. Wir behalten sie bei, nicht nur wegen des zur Verfügung stehenden Raums, sondern gleichermaßen in Besorgnis über eine fortschreitende Auszehrung des UtopieBegriffs.

Der Hauptakzent der Darstellung trifft wieder die deutsche und englische Utopie. Es muß verständlich sein, daß es für den Einzelnen, je näher die Darstellung der Gegenwart rückt, immer schwerer wird, die Utopie international im Blick zu behalten. Wir können daher nur in einer schlaglichtartigen Betrachtungsweise versuchen, ein wenig von der Schuld anderen Nationalliteraturen gegenüber abzutragen; auch dabei ist unvermeidbar, daß wichtige Texte nicht erwähnt werden. Die Kurzinterpretationen zur deutschen Utopie wurden um zwei Texte vermehrt; das Kapitel zur Science Fiction wurde völlig neu geschrieben; der pädagogische Exkurs am Schluß der ersten Auflage wurde, da er sich zu subjektiv gestaltet hätte, in eine Auswahlbibliographie umgewandelt.

Kiel, im Oktober 1981

Wolfgang Biesterfeld 


\section{ABKüRZUngen}

ABG Archiv für Begriffsgeschichte

ARW Archiv für Religionswissenschaft

BE Bildung und Erziehung

BfD Blätter für den Deutschlehrer

BNYPL Bulletin of the New York Public Library

CQ Classical Quarterly

DD Diskussion Deutsch

DVjs Deutsche Vierteljahrsschrift für Literaturwissenschaft und Geistesgeschichte

DU Der Deutschunterricht

Extr. Extrapolation

GLL German Life and Letters

GRM Germanisch-Romanische Monatsschrift

JHI Journal of the History of Ideas

MLR Modern Language Review

NS Die Neueren Sprachen

PLMA Publications of the Modern Language Association of America

PQ Philological Quarterly

PR Pädagogische Rundschau

QR Quarterly Review

SG Studium Generale

$\mathrm{Slg} \quad$ Die Sammlung

SM Sammlung Metzler

TAPhA Transactions of the American Philological Association

TCL Twentieth Century Literature

TSLL Texas Studies in Literature and Language

ZAA Zeitschrift für Anglistik und Amerikanistik

$\mathrm{ZfdPh} \quad$ Zeitschrift für deutsche Philologie

ZRGG Zeitschrift für Religions- und Geistesgeschichte 


\section{VERZEICHNIS DER WICHTIGSTEN LITERATUR*}

Forschungsberichte:

Karl Reichert: Utopie und Staatsroman. Ein Forschungsbericht. In: DVjs 39 (1965), S. 259-287.

Hinrich Hudde: Die literarische Gattung Utopie. Forschungsbericht unter besonderer Berücksichtigung der französischen Literatur. In: Romanist. Zeitschr. f. Literaturgesch. 1 (1977), S. 132-143.

\section{Kompendium:}

Michael Winter: Compendium Utopiarum. Typologie und Bibliographie literarischer Utopien. Bd. 1. Von der Antike bis zur deutschen Frühaufklärung. 1978 (Bibl.).

Bibliographien (Quellen):

Ludwig Hevesi: Katalog einer merkwürdigen Sammlung von Werken utopistischen Inhalts (16.-20. Jahrhundert). Einleitung von Friedrich Kleinwächter. Wien 1912.

George Peabody Gooch: Bibliography of political theory. London 1916.

B. M. Headicar u. C. Fuller: "Utopias«. In: London Bibliography of Social Sciences. Bd. 3. London 1931, S. 1104-1106.

Rita Falke: Versuch einer Bibliographie der Utopien. In: Romanist. Jb. 6 (1953/54), S. 92-109.

Everett Franklin Bleiler: A Check-List of Fantastic Literature. A Bibliography of Fantasy, Weird, and Science Fiction in the English Language. Chicago 1948.

Heinz Bingenheimer: Katalog der deutschsprachigen utopisch-phantastischen Literatur. 1460-1960. 1960.

Ignatius Frederick Clarke: The tale of the future from the beginning to the present day. A checklist of those satires, ideal states, imaginary wars and invasions, political warnings and forecasts, interplanetary voyages and scientific romances - all located in an imaginary future period - that have been published in the United Kingdom between 1644 and 1960. London 1961.

Regis Messac: Esquisse d'une chronobibliographie des »utopies«. Lausanne 1962.

Jakob Bleymehl: Beiträge zur Geschichte und Bibliographie der utopischen und phantastischen Literatur. 1965.

Pierre Versins: Encyclopédie de l'utopie, des voyages extraordinaires et de la science fiction. Lausanne 1972.

Utopia e fantascienza (Pubblicazioni del istituto di anglistica, Università di Torino). Turin 1975.

* Titel, die umfangreichere Bibliographien der Primär- und/oder Sekundärliteratur enthalten, sind im gesamten Band mit "(Bibl.)« gekennzeichnet. 
Glenn Robert Negley: Utopian Literature: A Bibliography with a Supplementary Listing of Works Influential in Utopian Thought. Lawrence (Kansas) 1977.

Lyman Tower Sargent: British and American utopian literature, 1516-1975. An annotated bibliography. Boston (Mass.) 1979.

Anthologien:

Voyages imaginaires, songes, visions et romans cabalistiques. Hg. v. Thomas Garnier. 39 Bde. Amsterdam (Paris!) 1787-1789.

Utopias del renacimiento. Hg. v. Eugenio Imaz. México 1941.

Utopisti italiani. Hg. v. Carlo Curzio. Rom 1944.

The Quest for Utopia. An Anthology of Imaginary Societies. Hg. v. Glenn Negley u. Max J. Patrick. New York 1952.

Der utopische Staat (Morus, Campanella, Bacon). Hg. v. Klaus J. Heinisch. 1960. (Bibl.).

Reise nach Utopia. Französische Utopien aus drei Jahrhunderten. Hg. v. Werner Krauss. 1964. (Bibl.).

Reisen nach Nirgendwo. Ein geographisches Lügengarn aus vielerlei fremden Fäden zusammengesponnen. Hg. v. Jürgen Dabl. 1965.

Utopischer Mond. Mondreisen aus drei Jahrtausenden. Hg. v. Kurt Jaritz. Wien 1965.

French Utopias. An anthology of ideal societies. Hg. v. Frank E. Manuel u. Fritzi P. Manuel. New York u. London 1966.

Phantastische Raumfahrt. Erzählungen der Weltliteratur. Hg. v. Heinar Köbl (= (Goldmann-Tb. 2680). München o. J.

Utopian literature. A Selection. Hg. v. J. W. Jobnson. New York 1968.

Dichter reisen zum Mond. Utopische Reiseberichte aus zwei Jahrtausenden. Hg. v. Helmut Swoboda. 1969.

Willkommen auf dem Mars. Berichte vom Leben auf anderen Planeten. Hg. v. Helmut Swoboda. 1970.

Outrepart: Anthologie d'utopies, de voyages extraordinaires, et de science fiction, autrement dit, de conjectures rationelles. Hg. v. Pierre Versins. Paris 1971.

Der Traum vom besten Staat. Texte aus Utopien von Platon bis Morris. Hg. v. Helmut Swoboda. 1972.

Utopies au siècle des lumières. Hg. v. Albert Soboul. Paris 1972.

Anthologie zur Begriffsgeschichte:

Utopie. Begriff und Phänomen des Utopischen. Hg. v. Arnhelm Neusüss. 1968.

Bibliographie (Sekundärliteratur):

Gorman Beauchamp: Themes and uses of fictional utopias: a bibliography of secundary works in English. In: Science Fiction Studies 4 (1977), S. 55-63. 


\section{Sammelbände zur Forschung:}

Vom Sinn der Utopie. Eranos-Jb. 1963. Zürich 1964.

Utopias and Utopian Thought. Hg. v. Frank E. Manuel. Boston u. Cambridge 1966 (Dt.: Wunschtraum und Experiment. Vom Nutzen und Nachteil utopischen Denkens. 1970).

Säkularisation und Utopie. Ebracher Studien. Ernst Forsthoff z. 65. Geb. 1967.

Der utopische Roman. Hg. v. Rudolf Villgradter u. Friedrich Krey. 1973.

Deutsches utopisches Denken im 20. Jahrhundert. Hg. v. Reinhold Grimm u. Jost Hermand. 1974.

Studi sull' utopia. Hg. v. Luigi Firpo. Florenz 1977.

Themenhefte von Zeitschriften:

Kursbuch 14 (1968).

Sozialistische Zeitschr. f. Kunst u. Gesellschaft 18/19 (1973).

Kürbiskern 1 (1975).

Allgemeine Darstellungen:

Andreas Voigt: Die sozialen Utopien. Fünf Vorträge. 1906.

Rudolf Helm: Utopia. Gedenkrede zur Jahresfeier der Universität Rostock. 1921.

Joyce Oramel Hertzler: The History of Utopian Thought. London 1923.

Lewis Mumford: The Story of Utopias. Ideal Commonwealths and Social Myths. London etc. 1923.

Paul Bloomfield: Imaginary Worlds, or, The Evolution of Utopia. London 1932.

Georg Quabbe: Das letzte Reich. Wandel und Wesen der Utopie. 1933.

Hans Freyer: Die politische Insel. Eine Geschichte der Utopien von Platon bis zur Gegenwart. 1936.

Wolf-Dietrich Müller: Geschichte der Utopia-Romane der Weltliteratur. 1938. (Bibl.)

Harry Ross: Utopias old and new. London 1938. (Bibl.)

James Osler Bailey: Pilgrims through space and time. Trends and patterns in scientific and utopian fiction. New York 1947. (Bibl.)

Heinrich Rodenstein: Die Utopisten. 1949.

Marie Louise Berneri: Journey through Utopia. London 1950. (Bibl.)

Raymond Ruyer: L'Utopie et les utopistes. Paris 1950. (Bibl.)

John Atkins: Tomorrow Revealed. New York 1956.

Ugo Fedeli: Un viaggio alle sIsole Utopiar. Ivrea 1958.

René Dubos: The Dreams of reason. New York 1961.

Franz Baumer: Paradiese der Zukunft. Die Menschheitsträume vom besseren Leben. 1967.

Jean Servier: Histoire de l'Utopie. Paris 1967 (Dt.: Der Traum von der großen Harmonie. Eine Geschichte der Utopie. 1971).

Walter Harry Green Armytage: Yesterday's Tomorrows. A Historical Survey of Future Societies. London 1968.

George Kateb: Utopia. New York 1971. 
Ferdinand Seibt: Utopica. Modelle totaler Sozialplanung. 1972.

Helmut Swoboda: Utopia. Geschichte der Sehnsucht nach einer besseren Welt. Wien 1972.

Raymond Trousson: Voyages aux pays de nulle part. Histoire littéraire de la pensée utopique. Brüssel 1975. (Bibl.)

Ian Todd u. Michael Wheeler: Utopia: An Illustrated History. New York 1978.

Ignatius Frederick Clarke: The Pattern of Expectation, 1644-2001. London 1979.

Frank E. Manuel u. Fritzi P. Manuel: Utopian Thought in the Western World. Cambridge (Mass.) 1979.

Darstellungen einzelner Aspekte - kritische Darstellungen:

Eugen Heinrich Schmitt: Der Idealstaat. 1904.

Friedrich Fürst $v$. Wrede: Die Entwicklung des Staatsromans. In: Deutsche Revue 30 (1905), S. 141-156.

Paget Violet: On Modern Utopias. London 1908.

Rudolf Blüber: Moderne Utopien. Ein Beitrag zur Geschichte des Sozialismus. 1920.

Hans Freyer: Das Problem der Utopie. In: Deutsche Rundschau 183 (1920), S. 321-345.

Hans Müblestein: Über die Rolle des Utopismus im Sozialismus, Anarchismus und Kommunismus der neueren Zeit. 1922.

Karl Schmückle: Logisch-historische Elemente der Utopie. 1923.

Alfred Doren: Wunschräume und Wunschzeiten. In: Vortr. d. Bibl. Warburg 4 (1927), S. 158-205.

Alphons Maluschka: Technik, Dichtung, Utopie. Wien 1927.

Fernando Vida Najera: Estudios sobre el concepto y la organización del estado en las »utopías «. Madrid 1928.

Sergius Hessen: Der Zusammenbruch des Utopismus. In: Festschr. Th. G. Masaryk z. 70. Geb. Bd. 1. 1930.

Frances Theresa Russell: Touring Utopia. The Realm of Constructive Humanism. New York 1932.

René-Louis Duyon: Variations de l'»utopie«. Paris 1933.

Hilda Diana Oakeley: The False State. London 1937.

Barna Horváth: Der Sinn der Utopie. In: Zeitschr. f. öffentl. Recht 20 (1940), S. 198-230.

Gerhard Ritter: Machtstaat und Utopie. Vom Streit um die Dämonie der Macht seit Macchiavelli und Morus. 1940.

Theodor Bübler: Von der Utopie zum Sozialstaat. Randbemerkungen zu einem zeitgemäßgen Problem. 1942.

Pierre Paraf: Les cités du bonheur. Paris 1945.

Ernst Bloch: Freiheit und Ordnung. Abriß der Sozialutopien. New York 1946.

Arthur Ernest Morgan: Nowhere was Somewhere. How History makes Utopias and how Utopias make History. Chapel Hill 1946.

Friedrich Knapp: Über den utopischen Roman. In: Welt und Wort 3 (1948), S. 284-286. 
Adolf Grabowsky: Die politischen Utopien und ihre Probleme. In: Schweizer Rundschau NF 48 (1948/49), S. 89-100.

Ernst Cassirer: Vom Mythus des Staates. Zürich 1949.

Erich Kabler: Die Wirklichkeit der Utopie. 1949.

Ernest Lee Tuveson: Millennium and Utopia. A Study in the Background of the Idea of Progress. Berkeley u. Los Angeles 1949.

Erich Bliesener: Zum Begriff der Utopie. Diss. (Masch.) Frankfurt a.M. 1950.

Max von Brück: Der Untergang der Utopie. In: Die Gegenwart 5 (1950), S. 13-15.

Martin Buber: Pfade in Utopia. 1950.

Wolfgang Pfeiffer-Belli: Utopia - einst und heute. In: Die Begegnung 5 (1950), S. 75-76.

Paul Roth: Utopien als Spiegelbilder ihrer Zeit. In: Stimmen der Zeit 148 (1950/51), S. 43-53.

Alfred Däubler: Die Utopie als Denkform. Diss. Tübingen 1951.

Helmut Kern: Staatsutopie und allgemeine Staatslehre. Ein Beitrag zur allgemeinen Staatslehre unter besonderer Berücksichtigung von Thomas Morus und H. G. Wells. Diss. Mainz 1951.

José Ortega y Gasset: Vom Menschen als utopischem Wesen. 1951.

Paul Tillich: Politische Bedeutung der Utopie im Leben der Völker. 1951.

Rita Falke: Persönliche Freiheit und die Utopien. Diss. (Masch.) Hamburg 1954.

Ludwig Marcuse: Vom Wesen der Utopie. 1952.

Rita Falke: Utopie - logische Konstruktion und chimère. Ein Begriffswandel. In: GRM 27, NF 6 (1956), S. 76-81.

Franz Altheim: Utopie und Wirtschaft. Eine geschichtliche Betrachtung. 1957.

Martin Schwonke: Vom Staatsroman zur Science Fiction. Eine Untersuchung über Geschichte und Funktion der naturwissenschaftlich-technischen Utopie. 1957. (Bibl.)

Wolfgang R. Heilmann: Die Sozialutopien und der Sozialutopismus. Diss. Tübingen 1959.

Roger Mucchielli: Le mythe de la cité idéale. Paris 1960.

Frederick Lodewijk Polak: De toekomst is verleden tijd. Leiden 1960.

Sergio Sarti: Utopismo e mondo moderno. Palermo 1960.

R. B. Schmerl: Reason's dream. Anti-totalitarian themes and techniques of fantasy. Diss. Un. of Michigan 1960.

Hubertus Schulte Herbrüggen: Utopie und Anti-Utopie. Von der Strukturanalyse zur Strukturtypologie. 1960. (Bibl.)

Georges Duveau: Sociologie de l'utopie. Paris 1961.

Horst Helmut Kaiser: Subjekt und Gesellschaft. Studie zum Begriff der Utopie. Diss. Frankfurt a. M. 1960.

Thomas Nipperdey: Die Funktion der Utopie im politischen Denken der Neuzeit. In: Archiv f. Kulturgeschichte 44 (1962), S. 357-378.

Chad Walsh: From Utopia to nightmare. London 1962.

George Kateb: Utopia and its enemies. London 1963. 
Erich Reigrotzki: Die Utopialität als wissenschaftliche Kategorie. In: Sozialwissenschaft u. Gesellschaftsgestaltung. Festschr. Gerhard Weisser. 1963, S. 103-119.

Giorgio Uscatescu: Utopía y plenitud histórica. Madrid 1963.

Hans Gerd Rötzer: Utopie und Gegenutopie. In: Stimmen der Zeit 89 (1963/64), S. 356-365.

E. M. Cioran: Geschichte und Utopie. 1965.

Nortbrop Frye: Varieties of literary utopias. In: Daedalus 94 (1965), S. 323-347.

Frank E. Manuel: Toward a Psychological History of Utopias. In: Daedalus 94 (1965), S. 293-322.

Jean Meynaud: Les spéculations sur l'avenir. Montreal 1965.

Jean-Jacques Chevallier: Denker, Planer, Utopisten. Die großen politischen Ideen. 1966.

Constantinos Apostolou Doxiadis: Between Dystopia and Utopia. London 1966.

Horst Brunner: Die poetische Insel. Inseln und Inselvorstellungen in der deutschen Literatur. Diss. Erlangen-Nürnberg 1967.

Moses J. Finley: Utopianism Ancient and Modern. In: The Critical Spirit. Essays in Honour of Herbert Marcuse. Boston 1967, S. 3-20.

Claude-Gilbert Dubois: Problèmes de l'utopie. Paris 1968.

Lars Gustafsson: Utopier och andra essäer om >dikt` och >liv`. Stockholm 1969. (Dt.: Utopien. Essays. 1970.)

Robert C. Elliott: The Shape of Utopia. Studies in a literary genre. Chicago 1970.

Robert Nozik: Anarchie, Staat, Utopia. München o.J.

Dominique Desanti: Les socialistes de l'utopie. Paris 1971.

Jobn T. Wilson: Is Utopia Possible? In: English 20 (1971), S. 51-55.

Alexandre Cioranescu: L'avenir du passé. Utopie et littérature. Paris 1972.

Robert Heiss: Utopie und Revolution. Ein Beitrag zur Geschichte des fortschrittlichen Denkens. 1973.

Gerhard Friedrich: Utopie und Reich Gottes. Zur Motivation politischen Verhaltens. 1974.

Elisabeth Hansoth: Perfection and progress: Two modes of utopian thought. Cambridge (Mass.) 1974.

Hans-Georg Soeffner: Der geplante Mythos. Untersuchungen zur Struktur und Wirkungsbedingung der Utopie. 1974 (recte: 1975).

Bernardo Cattarinussi: Utopia e società. Mailand 1976.

W. K. Thomas: The underside of utopias. In: College English 38 (1976/77), S. 356-372.

Christian Enzensberger: Die Grenzen der literarischen Utopie. In: Akzente 28 (1981), S. 44-60.

Jost Hermand: Orte. Irgendwo. Formen utopischen Denkens. 1981. 